Vol. 19, n² | 2015

Varia

\title{
La publicité de la police dans la ville de Paris dans la deuxième moitié du XVIII ${ }^{\mathrm{e}}$ siècle
}

\section{Nicolas Vidoni}

\section{OpenEdition}

\section{Journals}

Édition électronique

URL : http://journals.openedition.org/chs/1602

DOI : 10.4000/chs. 1602

ISSN : 1663-4837

Éditeur

Librairie Droz

Édition imprimée

Date de publication : 1 novembre 2015

Pagination : $93-118$

ISBN : 978-2-600-01908-8

ISSN : $1422-0857$

\section{Référence électronique}

Nicolas Vidoni, « La publicité de la police dans la ville de Paris dans la deuxième moitié du XVIIIe siècle », Crime, Histoire \& Sociétés / Crime, History \& Societies [En ligne], Vol. 19, n² | 2015, mis en ligne le 01 novembre 2017, consulté le 04 mai 2019. URL : http://journals.openedition.org/chs/1602 ; DOI : 10.4000/chs. 1602 


\title{
La publicité de la police dans la ville de Paris dans la deuxième moitié du XVIIIe siècle
}

\author{
Nicolas Vidoni ${ }^{1}$
}

\begin{abstract}
La police, entendue comme une pratique, s'offrait à la vue des Parisiens et exigeait une visibilité.

L'action des agents de la Lieutenance générale de police, sur le terrain, nécessitait parfois une prise de parole. Le XVIII e siècle vit ces prises de parole s'enrichir, et être diffusées au "public», par l'écrit. Cette diffusion visait à expliquer les nouvelles normes et justifier les nouveaux modes d'agir des policiers.

La Lieutenance mobilisa les savoirs urbains de son temps pour rationaliser son action. Elle utilisa alors la médiation et la médiatisation des institutions scientifiques, qui entraînèrent un regain de publicité de son action.

En retour, elle suscita des commentaires qui, diffusés, produisirent un débat sur la ville et sa police, que la Lieutenance souhaitait éviter, mais dont elle dut tenir compte. Cela déboucha, paradoxalement, sur une mise en question de l'action policière qui sortit ainsi du «secret».
\end{abstract}

Early-modern policing, as a practice, presented itself to the Parisians in a manner that was intended to be highly visible.

The activities on the ground by the members of the police force of the Parisian Lieutenant général sometimes required directives to the public. During the 18th century these directives multiplied and were circulated in writing to the 'public'. These directives aimed at explaining new standards and to justify new approaches by the police.

The Paris police authorities mobilised its knowledge about the city in order to justify its actions. They employed the communication skills of scientific institutions as well as their experience of dissemination of information, which in turn led to better public justification for its actions.

In return, the authorities received suggestions from the public which, when published, generated further debate about the city and its police. This was a debate which the authorities avoided getting directly involved in, but, at the same time, had to take into account. Paradoxically this resulted in questioning policing itself, which thereby could no longer remain in obscurity.

\footnotetext{
Nicolas Vidoni est titulaire d'un CAPES d'histoire-géographie (2004) et d'un doctorat (2011), PRCE à Aix-Marseille, Université Paris I. Chercheur du laboratoire Telemme, MMSH, Aix-en-Provence. Ses principales publications : Résister à la police: y a-t-il une spécificité féminine ?, in Gonzalez-Raymond, A. (dir.), Résister, Montpellier, Presses Universitaires de la Méditerranée, 2011, pp. 370-388; Une «police des Lumières»? La «violence» des agents de police à Paris au milieu du XVIII ${ }^{e}$ siècle, Rives méditerranéennes, 2011, 40, pp. 43-66, revue en ligne [rives.revues.org]; Les violences policières contre les femmes à Montpellier au milieu du XVIII ${ }^{\mathrm{e}}$ siècle, in Faggion, L. Regina, C. (dir.), La violence. Regards croisés sur une réalité plurielle, CNRS éditions, Paris, 2010, pp. 349-365.
} 
u commencement de la Révolution parut un ouvrage virulent de dénonciation de la police parisienne effectuée par l'ancienne Lieutenance générale, qui contribua à monumentaliser la «légende noire» de cette police ${ }^{2}$. Fruit des observations du ci-devant agent de la Lieutenance Manuel, elle livrait au public, par le moyen de scènes savamment racontées et en levant le voile qui recouvrait l'institution, les secrets de la police, en particulier les malversations, les concussions, les pratiques illégales et illégitimes, en bref l'arbitraire d'un groupe d'hommes plus préoccupés de leur intérêt propre que de l'intérêt du public ${ }^{3}$.

En mettant sur la place publique les pratiques policières et la police de la monarchie, l'ouvrage prétendait trancher avec les conceptions administratives qui faisaient de cette activité étatique un domaine secret. Pourtant, dans les faits, la police était sous le regard de l'opinion publique depuis quelques décennies ${ }^{4}$. Manuel fit donc preuve de partialité en présentant cette police de la capitale comme une activité reposant sur le secret. Le thème du rapport de la police au public, voire à la sphère publique, peut être approfondi par l'étude de la publicité de l'action policière de la Lieutenance 5 . Dans le contexte de la deuxième moitié du XVIII ${ }^{e}$ siècle, au cours de laquelle «l'opinion publique $»^{6}$ ou le public devint un interlocuteur de la monarchie administrative ${ }^{7}$, ce rapport évolua en resserrant les liens entre public et institution policière. Les rapports entre société et État furent en effet modifiés, et orientèrent les pratiques de la monarchie absolue vers un certain libéralisme, inégal en intensité selon les domaines et au fil des ans, mais malgré tout bien réel, qui devait tenir compte du public.

La police, elle, doit être dégagée de la seule sphère du droit et de l'étude des règlements, et étudiée comme une pratique ${ }^{8}$. Insérée très fortement dans l'espace urbain, elle ne peut se déprendre de son inscription matérielle et de son insertion sociale dans la population. Par ailleurs, la Lieutenance, tout au long du siècle des Lumières, mit en œuvre des pratiques nouvelles de contrôle de l'espace et des populations, qui se détachèrent largement des formes traditionnelles de règlement des conflits et des désordres urbains ${ }^{9}$. Plus professionnelle, bureaucratique ${ }^{10}$, et initiée sur le terrain par des agents plus mobiles, elle tendit à se déprendre de l'insertion personnelle de chaque officier dans son espace quotidien d'action, sans pour autant que tous eussent appliqué ces nouvelles méthodes ${ }^{11}$.

2 Manuel (1792). Cet article a été rédigé dans le cadre du programme ANR «Syspoe» $\mathrm{n}^{\circ}$ ANR-12BSH3-0004.

3 Dès son propos liminaire intitulé «Aux Amis de la Constitution», Manuel écrivait: «Il m'a semblé que c'étoit entrer dans ses vues [au peuple] comme dans ses besoins, que de lui révéler toutes les turpitudes de cette police qui n'a jamais rien trouvé d'impossible, parce que pour elle il n'y eut jamais rien d'injuste ni de malhonnête», ibid., t. 1, p. 5.

4 Brouillet (2005, pp. 169-190).

5 La bibliographie est très abondante sur le thème de «l'espace public». Nous renverrons, pour des travaux fondateurs et d'autres critiques, à Habermas (1978); Baker (1987); Boucheron, Offenstadt (2011).

6 Baker (1987).

7 Roche (1998, chap. IX et XIV).

8 Napoli (2003).

9 Nous nous permettons de renvoyer à Vidoni (2011).

10 Milliot (2011).

11 Berlière (2012). Pour la question des modifications des systèmes et des styles de police en France et en Europe, Milliot (2006); Denys et al. (2009). 
Cette déconnexion impliqua de nouveaux modes de légitimation et d'acceptation par la population de l'action policière. Deux axes ont été mis en lumière par l'historiographie à la suite de la sociologie, celui de la rencontre avec un «besoin » de sécurité (lui-même né pour partie de la création d'un sentiment d'insécurité par les autorités monarchiques dès le $\mathrm{XVI}^{\mathrm{e}}$ siècle ${ }^{12}$ ), et celui de l'usage de la violence qui, par le biais de crises et de remises en cause de l'action physique de la police, ont conduit à définir les formes acceptables d'action policière dans la ville ${ }^{13}$. Un troisième axe peut être appréhendé, qui est celui d'une publicisation croissante de certaines activités policières par la Lieutenance elle-même. Loin de se réduire à une simple «propagande», elle fit naître une réflexion dans le public sur ce qu'était la police. Une dialectique se construisit ainsi, plutôt qu'un dialogue ou une «négociation $»^{14}$, entre deux entités qui étaient liées, à rebours de l'image traditionnelle et stricte du secret d'État découlant de la raison d'État ${ }^{15}$.

Les liens entre «public» et Lieutenance générale se tissèrent par trois grandes voies. La première fut celle de la publicisation de l'action policière par des moyens classiques, telles que les cérémonies, ou par une prise nouvelle de parole en public. Le deuxième passa par l'intégration de personnalités scientifiques éminentes et par la mobilisation de la population pour l'action même de la police, modifiant du même coup sa mise en œuvre et sa réception par le public. La dernière, bien que les trois fussent intriquées, fut la mobilisation des réseaux médiatiques par la Lieutenance, certes pour promouvoir son action, mais encore pour légitimer aux yeux de l'opinion publique certaines pratiques policières tout en en taisant certaines.

\section{LA POLICE FACE AUX PUBLICS DANS LA SECONDE MOITIÉ DU XVIIIE SIÈCLE}

La Lieutenance de police, dès sa création en 1667, s'est adressée à la population, en recourant à des procédés classiques et communs à toutes les institutions urbaines. Par les voies de l'affichage et des cris publics, ainsi que par l'activité médiatrice quotidienne des commissaires du Châtelet dans leur quartier, elle divulguait et explicitait les normes comportementales et d'usage de l'espace urbain auprès du public parisien ${ }^{16}$. De plus, dans les rues, l'action des agents de la Lieutenance se faisait sous l'œil et à la vue des personnes présentes, qui constituaient ainsi un public plus ou moins impliqué. À côté de ces situations quotidiennes, des «moments» ont eu pour but d'intégrer la population à l'action policière. Au quotidien, les officiers de police parlaient aux individus qu'ils administraient. Mais à côté de ce procédé classique, les agents de la Lieutenance ont été amenés, plus fréquemment à partir du milieu du siècle, à prendre publiquement la parole à une échelle plus élevée. De plus, des cérémonies urbaines, en particulier les ouvertures de marché, ont contribué à mettre en exergue l'institution policière.

\footnotetext{
Roussel (2013).

13 Jobard (2002); Farge, Revel (1988); Moreau de Bellaing (2003).

14 Bastien (2006).

15 Thuau (2000); Lazzeri, Reynié (1992).

16 Piasenza (1990); Roussel (2012); Offenstadt (2013).
} 


\section{La prise de parole policière}

La prise de parole policière s'est effectuée à plusieurs échelles dans la ville. La première et la plus fréquente, est celle qui concernait les acteurs policiers au quotidien. Le commissaire et l'inspecteur, opérant dans leur quartier, prenaient la parole devant les foules ou les individus, et selon différents registres et modes opératoires ${ }^{17}$. Les archives ont ainsi conservé les actes du «cours ordinaire des choses» et les actions plus éclatantes de ces agents ${ }^{18}$. Le but, semble-t-il, était pourtant souvent le même: apaiser les tensions interindividuelles qui risquaient de déboucher sur des situations conflictuelles collectives.

Ainsi, au moment des émeutes liées aux rumeurs des enlèvements d'enfants (1750-1752), les inspecteurs de police, ainsi que leurs hommes de main, ont été sévèrement contestés pour leurs méthodes violentes par une partie de la population parisienne ${ }^{19}$. La remise en cause de la brutalité et de l'arbitraire de ces agents bien différents des commissaires, a entraîné chez eux une prise de conscience de la nécessité de modérer certains faits et gestes pourtant usuels et usités. On le comprend lorsque l'on étudie les archives de l'inspecteur Poussot, chargé de surveiller l'espace urbain et d'appréhender les contrevenants lors de patrouilles diurnes et nocturnes. Obligé d'interrompre ses patrouilles pendant deux mois l'été, il reprend calmement cette activité en veillant à ce que ses exécutants restent doux et modérés dans leurs paroles et leurs actes. Il l'écrit dans son rapport au Lieutenant Général de Police Berryer le 30 octobre 1750, à propos d'une altercation que ses agents ont eue avec des individus contrôlés la nuit, et qu'il qualifie de «sédition». Après avoir parlé aux uns et aux autres, il fait «beaucoup de peur et point de mal» à certains, et apaise les autres par ses propos. Il couche alors sur le papier ces réflexions sur ses agents:

Le Sr Roullier et Bouton se sont comportés avec toute la sagesse et la prudence possible je puis vous asseurer M. que je suis extremement sensible a de pareille scenes quoi que personne de nous n'eut tort sinon trop de vivacité de la part de M. Ferry, mais il s'est trouvé fort embarassé car l'homme qu'il tenoit, la pris pareillement par le Colet, il y avoit encore 4 ou 5 femmes autour de lui, moi qui crioit a tout le monde Mrs ne frappés pas, j'ay grondé le Sr Ferry un peu aigrement en lui disant qu'il faloit éviter de pareilles scenes et que la première force que nous devions employer étoit la douceur et la raison, il ma repondu quil n'étoit pas aisé à un honnete homme de se voir insulté ${ }^{20}$.

L'intervention de l'inspecteur, ici responsable de l'opération, ne vise nullement à remettre en cause la réaction de ses agents, mais plutôt à montrer les changements dans son propre comportement face aux actes violents qui risquent, comme cela se produit fréquemment, d'ameuter la foule et d'entraîner des contestations qui peuvent dégénérer. L'insistance sur la modération est le trait distinctif de ce nouvel ethos policier, qui a parcouru les comportements des agents sur le terrain et les directives des Lieutenants généraux successifs qui, soit dit en passant, ont toujours analysé les

Les commissaires opéraient un magistère régulateur. Sur cette question, Farge (1986); Berlière (2012).

18 Farge (1986).

19 Farge, Revel (1988); Piasenza (1987).

20 Bibliothèque nationale de France (désormais BnF), Arsenal, manuscrit (désormais ms) 10136, p. 575. 
réactions collectives à l'action policière en termes de «sédition» ou de contestation dangereuse.

L'attention soutenue portée à la conduite des agents dans l'espace public a été systématisée par Sartine (1759-1774) et poursuivie par Lenoir (1774, 1776-1785), ce dont rend compte une lettre programmatique adressée aux syndics des inspecteurs par ce dernier en octobre 1774, au moment de son entrée en charge comme Lieutenant général de police ${ }^{21}$. Il écrit, d'un ton qui ne souffre aucune contestation, qu'il sera intransigeant sur les questions relatives aux procédures et aux actions policières. Les inspecteurs devront remplir les «fonctions» eux-mêmes, et ne pourront les «confi[er] arbitrairement à l'examen des commis et autres personnes [qui leur sont] attachés ». De même, lorsque les inspecteurs seront requis par les commissaires du Châtelet, ils devront se présenter «en personne $»^{22}$. L'objectif est d'agir en «vérité», et de faire preuve de "probité et désintéressement», afin d'assurer le succès du service «de la Police de la Capitale». Cette politique de codification des pratiques, accompagnée d'un respect strict de la légalité des procédures, et de l'implication personnelle des officiers dans leurs missions, répondait à ce moment à une série de critiques adressées à l'endroit du personnel policier - spécialement les inspecteurs -, souvent considéré par une partie de l'opinion publique comme agissant avec arbitraire et selon des motifs plus personnels que «d'utilité publique ${ }^{23}$. On perçoit ici une première tentative pour mettre en correspondance l'idéal d'une force publique impartiale, ce qu' attendait une partie de la population, et les pratiques de terrain des officiers de police ${ }^{24}$.

À un niveau bien supérieur, les Lieutenants généraux ont également été impliqués dans les débats relatifs aux orientations politiques et économiques du siècle. Une des questions les plus débattues, et pour laquelle les responsables policiers ont dû s'exprimer dans l'espace public, a été celle de la liberté du commerce, en particulier des grains. La libéralisation du commerce des grains a été menée progressivement ${ }^{25}$. À partir des années 1750, de nombreuses publications orientèrent le débat sur la question de la liberté intérieure (au royaume) de ce commerce. Ces écrits largement diffusés, mettaient en cause la traditionnelle police des grains, qui laissait les autorités locales agir pour réguler, et même contrôler, l'approvisionnement des villes. Deux édits de mai 1763 et juillet 1764 autorisèrent la libre circulation, l'accès au marché à qui le désirait, et une relative ouverture sur l'étranger pour l'exportation en-deçà d'un certain prix (30 livres le setier de blé dans les ports). Des oppositions surgirent face à cette politique libérale, en particulier en 1765 et 1767, à l'occasion de mauvaises récoltes qui firent augmenter les prix. Les parlements, dans un premier temps favorables à la liberté, commencèrent à revoir leur position. Ce fut le cas lors d'une assemblée générale de police tenue le 28 novembre 1768 au Parlement de Paris $^{26}$. Ces assemblées, fréquentes, réunissaient les autorités de la ville (municipales, parlementaire, religieuses, de police) afin de coordonner les politiques policières et

\footnotetext{
$\overline{21}$ BnF, Arsenal, $\mathrm{ms} 10015$, fol. $671 \mathrm{r}^{\circ}-673 \mathrm{v}^{\circ}$.

22 Ibid., fol. $672 \mathrm{v}^{\circ}$.

23 Ibid., fol. $673 \mathrm{r}^{\circ}$.

24 Sur cette question, Couture (2013), thèse que nous n'avons malheureusement pas pu encore consulter.

25 Sur cette question, et dans une bibliographie abondante, nous utilisons Grenier (2007); Meyssonnier (1989); Kaplan (1988).

26 Archives Nationales (désormais AN), K 1022, pièce ${ }^{\circ}$ 14, 110 folios.
} 
ainsi éviter tout conflit de compétences ou des contestations sur le terrain. À cette occasion, le premier président, François d'Aligre, rappela les «loix très anciennes respectables » qui avaient cours avant 1763, et interpella l'auditoire sur la situation de «misère» due aux monopoles et aux «emmagasinages» qui avaient cours dans Paris, pourtant exclus des édits de 1763-1764. L'avocat général Joly de Fleury porta une attaque plus violente contre une «secte» (les physiocrates) qui opéra une «révolution » imitée du modèle anglais, en insistant sur les conséquences néfastes de l'exportation des grains sur le marché intérieur. Selon lui, la situation ne tournait pas encore à la disette en raison des soins de la police de Paris, capable de constituer des stocks pour approvisionner les marchés de la capitale, et ainsi prévenir une émeute. Il réclamait que les lieux de vente soient limités aux marchés urbains, placés sous le regard de la police, avec une restriction du nombre des négociants, enregistrés et connus de la police. Il demandait également la limitation de l'autorisation d'exportation à quatre ports du royaume. C'est alors que le Lieutenant général Sartine fut obligé d'exprimer publiquement son opinion. Il n'y avait là rien de véritablement nouveau, puisque depuis la création de la Lieutenance en 1667, des assemblées de police étaient réunies chaque année. La nouveauté tenait dans le fait que Sartine, de même que Joly de Fleury (qui travaillaient sur d'autres dossiers main dans la main) répondaient à une brochure anonyme répandue dans le public intitulée Avis aux honnêtes gens. L'ouvrage, écrit par l'abbé Beaudeau, était à la fois une apologie des physiocrates et de la liberté du commerce des grains portée par la loi, et une critique acerbe contre les pratiques policières qui entravaient l'application de cette loi et la rendaient caduque ${ }^{27}$. La mise en cause de la probité des officiers supérieurs de la monarchie appelait une mise au point. Sartine estimait que la circulation des grains était «conforme au droit naturel», tout en qualifiant de danger une liberté «absoluë et illimitée». Fort de cette position médiane, il appuyait les propositions restrictives de l'avocat général en voulant réserver (par des règlements de police, et non par une loi) la vente des grains aux habitants, aux boulangers et ensuite - seulement - aux marchands, afin d'éviter toute spéculation abusive. Il estimait en effet que les «spéculations en grand [étaient] souvent utiles au bien public»par la capacité de mobilisation des quantités dont disposaient certains marchands, mais qu'elles devaient être fortement contrôlées ${ }^{28}$. Pour cela, il souhaitait réduire l'exportation de grains, en abaissant le prix du setier à 24 livres dans les ports au-delà duquel toute exportation fût impossible. Il présentait, in fine, la situation parisienne sous un jour moins terrible que le propos du premier président, en n'oubliant pas de vanter le système policier parisien de surveillance des marchés. Il ne développait pas, à cet égard, une pensée totalement hostile à celle de Beaudeau, mais encadrait les échanges, à tout moment, par l'action des agents de police sur le terrain. Il adoptait de fait une position médiane entre celles des physiocrates et du Parlement, chacune à un bout de l'échelle libérale, en raison d'une volonté de développer ses réflexions à partir de la connaissance du terrain qu'il possédait.

Ainsi, de la rue aux sphères politico-administratives, la police tendit à s'exposer publiquement dans la seconde moitié du XVIII e siècle, avec un apogée au moment de la parution d'un écrit de Lenoir intitulé Détail sur quelques établissemens de la Ville de Paris, demandé par Sa Majesté Impériale la Reine de Hongrie, à M. Le Noir,

\footnotetext{
27 Beaudeau (1768).

28 AN, K 1022, fol. $24 \mathrm{r}^{\circ}-\mathrm{v}^{\circ}$.
} 
Conseiller d'État, Lieutenant-Général de Police ${ }^{29}$. Ce mémoire imprimé et diffusé complétait un premier mémoire rédigé par le commissaire Lemaire en réponse à un questionnaire de la cour de Vienne à propos de la police de Paris ${ }^{30}$. Dans ce mémoire resté confidentiel, le commissaire présentait en détail (et à son avantage) l'organisation institutionnelle et pratique de la police parisienne, en attribuant à la Lieutenance un rôle central. Dans le mémoire public de Lenoir, des thèmes différents et nouveaux étaient abordés, tels que la politique hospitalière menée par la Lieutenance (ouverture d'hospices, accueil des nouveau-nés, lutte contre les maladies endémiques, etc.), la lutte contre l'insalubrité urbaine et les risques en découlant (les glaces et le nettoyage des rues, les vidanges des fosses d'aisance, des puits, etc.) ou encore la politique de secours aux accidentés, aux noyés ou aux blessés de la ville de Paris. Loin de n'être qu'une politique de surveillance et d'organisation des flux urbains, la police se faisait dispensatrice de «soins» au peuple, en agissant dans et sur la ville. La publication de ce mémoire n'est pas anodine, puisque Lenoir répondait alors à une demande de la part du public éclairé très préoccupé des questions d'aménagement urbain $^{31}$. Il dépassait le simple échange entre autorités administratives européennes pour s'adresser en premier lieu au public français (et parisien), tout en érigeant la police de la Lieutenance au rang de modèle. Un véritable échange s'instaurait ainsi entre l'espace public et l'autorité policière, contrainte pour une part de répondre aux demandes de l'opinion publique ${ }^{32}$. Une traduction de ce dialogue plus ou moins contraint est perceptible dans les cérémonies urbaines policières.

\section{Les cérémonies urbaines policières}

La Lieutenance participait en tant que force de police aux cérémonies urbaines parisiennes $^{33}$. Elle le faisait notamment en contrôlant et en surveillant le bon déroulement des cérémonies publiques. Son action fut sensible dès le début du siècle, lorsque les commissaires furent chargés de contrôler les échafaudages érigés pour la préparation de réjouissances publiques ${ }^{34}$, à l'occasion de célébrations de victoires militaires ou lors de fêtes en l'honneur du monarque ou de naissances royales ${ }^{35}$. Mais une évolution apparut dans ces manifestations publiques à l'occasion de l'ouverture de nouveaux marchés. L'implication de la Lieutenance se fit en effet très forte dans l'élaboration des projets de nouvelles halles ou nouveaux marchés, au détriment de l'autorité de l'Hôtel de Ville, traditionnellement en charge de ces questions, et trouva sur le terrain une traduction visible par la population. Nous concentrerons notre propos sur l'ouverture de la nouvelle Halle de Paris dans les années 17601770, et les continuations de cette mise en scène à l'occasion des aménagements de

\footnotetext{
$\overline{29 \quad \text { Lenoir (1780). }}$.

30 Lemaire (1879). À ce propos, Kaplan, Milliot (2009).

31 Wittman (2009).

32 Dans ses Mémoires, Lenoir rapporte que le «public ne pouvait se refuser d'applaudir » à l'emploi de réverbères pour éclairer les rues, mais qu'en ce qui concernait le pavé de la ville, «le public de mon temps étoit plus exigeant et malveillant que le public des temps précédents ». Milliot (2011, pp. 580 et 588).

33 Sur les cérémonies urbaines, Fogel (1989).

34 BnF, ms fr. 21693, fol. $210 \mathrm{r}^{\circ} s q$.

35 BnF, JdF 318.
} 
cette nouvelle infrastructure, en mettant en lumière les spécificités apparues dans l'organisation des cérémonies.

Avant 1760, le quartier des Halles, cœur de la capitale, concentrait une multitude de petits marchés spécialisés, qui produisaient un désordre important par l'encombrement des rues, les saletés produites et les embarras consécutifs à la rencontre d'une foule d'acheteurs et de vendeurs. Les rapports de l'inspecteur Poussot, alors affecté à ce quartier, firent remonter les plaintes des habitants qui se manifestèrent par d'autres voies auprès des autorités urbaines ${ }^{36}$. L'inspecteur n'hésitait pas, dans ses rapports, à proposer l'aménagement d'une halle plus grande, qui aurait permis de concentrer sur un même lieu les offreurs, les demandeurs et les produits. L'œil de la police pouvait ainsi contrôler les flux, les échanges et les prix dans une unité de temps et d'action. Pour cela, un aménagement pertinent devait être mené, qui permettrait d'embrasser du regard ce monde effervescent. La Lieutenance pratiqua à cet égard une double politique. Elle s'impliqua fortement dans le projet d'aménagement d'une grande halle sur le terrain de l'hôtel de Soissons et la construction d'une gare sur la Seine qui permit de centraliser l'approvisionnement fluvial $^{37}$. Par ailleurs, la Lieutenance soutint la publication d'ouvrages expliquant par le détail cette opération, en particulier l'ouvrage de Maille Dussaussoy, Le Citoyen désintéresse $e^{38}$. Les travaux, débutés en mars 1763, s'achevèrent en 1765, mais ne furent pas complets, puisque la gare sur la Seine - incombant à la Ville ne fut pas construite ${ }^{39}$. La nouvelle halle fut pourtant un des plus grands chantiers urbanistiques du siècle ${ }^{40}$, et concrétisa les rêves policiers puisque la halle aux blés, de forme circulaire (ce qu'imposa le Lieutenant général ${ }^{41}$ ), permettait un contrôle strict des échanges, de même que les halles annexes, ouvertes aux rues principales et fermées pour concentrer les personnes agissant dans ces espaces.

L'ouverture de ce lieu ne passa pas inaperçue, et l'action de la Lieutenance fut largement vantée à cette occasion. Un rapport conservé dans les archives du Parlement mentionne en effet que «L'ouverture de la Halle a été faite le 12 janvier 1767 en vertu d'une ordonnance de Police du 30 décembre 1766 et le meme jour 12 Janvier M le Lieutenant general de Police, M le Procureur du Roy et le Commissaire du quartier en ont pris possession $»^{42}$. L'emprise de la Lieutenance est sensible dans ce document, et la cérémonie qui eut lieu consacra le rôle du Lieutenant face à un Hôtel de Ville incapable de mener à bien la construction de la gare sur la Seine. Le rapport ne mentionne pas la cérémonie en elle-même, mais l'on peut supposer qu'elle se déroula à l'instar de l'ouverture du marché Saint-Antoine le 5 avril 1781 par Lenoir. Au cours de celle-ci, le Lieutenant général vérifia les titres et les actes de création du marché, puis se rendit à la suite de la procession des clercs vers le marché. Il y retrouva des détachements du guet, accompagnés de «fifres, tambours, en la symphonie de leurs compagnies», le commissaire du quartier Crespy, les

\footnotetext{
36 BnF, Arsenal, ms 10141, PV du 3 avril 1756 (p. 109) et du 6 août 1758 (pp. 196-197).

37 C'est ce que mentionnent les lettres patentes du 25 novembre 1762, qui insistent grandement sur le rôle joué par le Lieutenant général de police, AN, H 2157 et BnF, Joly de Fleury 1425.

38 Dussausoy (1767).

39 AN, H 2157.

40 Harouel (1993).

41 BnF, JdF 1425, fol. $26 \mathrm{r}^{\circ}$.

42 AN, H 2157.
} 
syndics des inspecteurs de police, des huissiers à cheval et à verge du Châtelet. Les forces policières étaient ainsi réunies pour montrer l'étendue de la magistrature policière sur la ville. Une procession autour du marché fut conduite alternativement par les chants du clergé et la «symphonie» de la garde de Paris, puis Lenoir fit

faire lecture à haute et intelligible voix de [son] ordonnance du seize mars dernier et à l'instant l'ouverture dudit marché a été faitte par nos ordres au son des instruments, et au milieu d'un peuple considérable en notre présence celle du procureur du roy [...] et après laditte ouverture laditte procession s'est remise en marche pour s'en retourner et nous l'avons suivie jusqu'à l'abbaye St Antoine où nous sommes entrés ${ }^{43}$.

Pour cette dernière ouverture, c'est l'abbaye Saint-Antoine qui avait sollicité l'ouverture du marché. Lenoir, au cours de la cérémonie, contrôla la régularité de cette création, et manifesta physiquement son contrôle sur ce lieu en décrétant et organisant l'ouverture. La cérémonie plaçait ainsi au cœur de l'action monarchique le Lieutenant général de police, garant aux yeux de la population du pacte nourricier qui unissait le monarque à ses sujets. Institutionnellement, symboliquement et physiquement, le Lieutenant général et ses officiers encadraient et régulaient l'activité essentielle au cœur de la ville. D'un point de vue plus personnel, Lenoir effaçait par sa présence le souvenir de sa disgrâce des années 1775-1776, à la suite de la guerre des Farines, qui avait servi de prétexte à Turgot pour le remplacer à la tête de la Lieutenance par son fidèle Albert, plus libéral et passé par le Bureau du commerce au sein duquel il avait quelque peu lutté contre les mécanismes traditionnels de régulation et d'encadrement du marché des grains.

L'emprise policière sur les marchés fut renforcée au début des années 1780 par les aménagements que Lenoir imposa pour la nouvelle halle. Il mobilisa deux architectes, Legrand et Molinos, pour couvrir la Halle aux blés ${ }^{44}$. Après une expertise et la réalisation de quelques essais, les deux architectes couvrirent la halle d'un dôme de verre. Au-delà de la performance technique, une cérémonie vanta le nouvel aménagement, imaginé par les architectes sur le modèle des grands monuments de Rome ou d'Athènes, mais également pensé pour favoriser la diffusion de la lumière et la ventilation des lieux, dans un contexte intellectuel aériste ${ }^{45}$. La publicisation de l'action urbanistique policière s'accrut quelques mois plus tard quand, les travaux achevés, une cérémonie de réjouissances publiques fut organisée dans la Halle par Moreau, architecte du roi et de la Ville, pour fêter la paix. La cérémonie se déroula le 14 décembre 1783 , devant 7000 à 8000 personnes $^{46}$, mais après que le Bureau de Ville, en accord avec le Lieutenant général de police et l'action d'organisation des commissaires dans la halle et les rues adjacentes, eût décidé du déroulement des réjouissances ${ }^{47}$. Le processus d'aménagement a été accompagné d'une campagne de publicité par le Journal de Paris, sur laquelle nous reviendrons ensuite.

AN, Y 9500, PV de l'ouverture du marché Saint-Antoine.

44 Tout ce dossier se trouve à la Bibliothèque Historique de la Ville de Paris (BHVP), CP 4823.

45 Ibid., «Mémoire sur un Projet de couverture pour la cour de la Halle au Bled, proposé a Mr le Lieutenant général de Police par Mr Legrand et Molinos architectes» 1782.

46 Ibid., «Description des réjouissances données en la nouvelle halle le dimanche 14 décembre 1783 à l'occasion de la paix».

47 Ibid., Décision du Bureau de Ville du 19 novembre 1783. 
Finalement, l'action de la Lieutenance, que ce soit sur le terrain urbain parisien, ou dans les sphères administratives et politiques de la monarchie, se confronta de plus en plus au public et à ses exigences. Différents niveaux sociaux et d'implication de ce public apparaissent. Il y a en effet loin entre les écrits des économistes et la participation d'une foule anonyme à une réjouissance publique. Mais dans les deux cas, les Lieutenants tinrent compte de cette présence pour organiser et vanter leur action. Ils insistèrent notamment sur leur efficacité. Ces discours, sans mentionner les autres institutions, en particulier l'Hôtel de Ville, mettaient en avant la «capacité à agir $»^{48}$ de la Lieutenance et, à contre-jour, le caractère emprunté des politiques urbaines concurrentes. Cela se retrouve également dans la mobilisation des initiatives scientifiques individuelles et collectives par la Lieutenance en vue d'agir plus efficacement.

\section{L'ACTION CONJOINTE DE LA LIEUTENANCE ET DE PERSONNALITÉS PUBLIQUES}

La mobilisation du public par la Lieutenance générale ne se fit pas sous la seule contrainte et sous la pression de l'opinion générale. En effet, la Lieutenance mobilisa les initiatives personnelles et collectives en vue d'améliorer le service de la police. Cela est très sensible dans le cas des concours académiques et du soutien de la police à certaines initiatives scientifiques, ainsi que pour les marchés publics et les modalités de soumission des entrepreneurs candidats pour ces marchés.

\section{Les concours}

La volonté d'améliorer le «service de la police» est manifeste chez tous les Lieutenants généraux de la seconde moitié du XVIII ${ }^{\mathrm{e}}$ siècle $^{49}$. Ils le firent selon des principes et des moyens différents, mais tous cherchèrent à rendre plus «efficace» cette action administrative. Ils le firent bien souvent en tentant d'améliorer l'espace urbain, emportés par le mouvement de «purification» de la ville, très net chez les aéristes ${ }^{50}$, les architectes et les faiseurs de projets urbains ${ }^{51}$.

Sartine le premier semble avoir compris l'intérêt des concours académiques pour multiplier l'audience des questions policières et parvenir à mobiliser un public plus large en vue de résoudre certaines difficultés essentiellement techniques ${ }^{52}$. Pour cela, il proposa pour 1763 un prix décerné par l'Académie des Sciences en vue d'améliorer l'éclairage urbain. Si le sujet n'était pas nouveau, la méthode l'était. Le Lieutenant général s'en remettait à la «sanction de l'Académie ${ }^{53}$ d'un point de vue scientifique et technique, cette dernière, en tant qu'institution capable de valider ou d'invalider des projets, étant chargée de se prononcer sur la faisabilité et le rapport

\footnotetext{
Mannoni (1994).

49 Milliot (2011, pp. 360-374).

50 Bourdelais (2001).

51 Lemas (2002 a et b).

52 Sur les concours et leur audience démultipliée, Cardona (2009).

53 Brian (1997).
} 
coût-avantages d'une invention technique ${ }^{54}$. Dans ce processus, le Lieutenant redoublait la sanction de l'Académie en validant ou invalidant d'un point de vue administratif, la réalisation et la faisabilité d'une invention, la faisant passer alors au rang d'innovation. Ce fut le cas pour les réverbères que le candidat Bourgeois de Chateaublanc proposa à l'Académie ${ }^{55}$. Son invention, jugée peu innovante (le procédé existait depuis $1744^{56}$ ), fut pourtant retenue par Sartine pour être testée dans les rues de Paris, sous l'œil de l'inspecteur de l'illumination Vanier. La combinaison d'un savoir scientifique et technique et d'un savoir pratique (celui de l'inspecteur) convainquit Sartine de retenir les réverbères pour équiper la ville.

Le successeur de Sartine, Lenoir, proclama constamment qu'il souhaitait poursuivre sur le chemin tracé par son prédécesseur ${ }^{57}$. Il y a là une certaine modestie de sa part, puisqu'il systématisa et amplifia ce procédé de recours aux institutions scientifiques. Il s'impliqua personnellement dans la création de plusieurs écoles (de Chimie, de Boulangerie) ainsi que dans la création de la Société Royale de Médecine, dirigée par son proche Vicq d'Azyr. Cette institution concurrença la Faculté de Médecine et l'Académie Royale des Sciences, parfois peu réactives et innovantes. Pour autant, Lenoir ne délaissa pas le moyen des concours, et fit proposer par la Société en 1778, un prix de 1200 livres pour l'année 1781 sur le sujet: «Déterminer quel peut être le meilleur traitement de la Rage $»^{58}$. Le concours ne permit pas de donner des mémoires satisfaisants. En 1783, lors de la séance publique du 11 mars, les membres de la Société regrettèrent que le prix, depuis cinq ans, n'eût pas trouvé de réponse intéressante, hormis le Traité sur la Rage de Andry (1780) tiré des premiers mémoires envoyés à la Société. Pour cette raison, un deuxième prix fut proposé par Lenoir, sur la question: «Déterminer quelles sont, parmi les maladies, soit aiguës, soit chroniques, celles qu'on doit regarder comme vraiment contagieuses; par quels moyens chacune de ces maladies se communique d'un individu à un autre, \& quels sont les procédés les plus sûrs pour arrêter les progrès de ces différentes contagions $»^{59}$. Le concours s' adressait particulièrement aux «Administrateurs \& aux Médecins », mieux à même de connaître les «précautions » à prendre dans les cas des «maladies auxquelles sont exposés les hommes rassemblés en grand nombre», dans les ateliers, les prisons, les hôpitaux. La mobilisation intellectuelle dépassait donc la stricte sphère des hommes de science, et incluait, par l'exposé des motifs, tous ceux qui s'intéressaient à la «chose publique». Le prix, comme le précédent, ne reçut pas de mémoire satisfaisant, du moins en 1785, et fut relancé ${ }^{60}$. Toutefois, Lenoir s'engagea publiquement à soutenir financièrement toute initiative qui permettrait d'apporter des «renseignements utiles ou des observations intéressantes». Cette fois, les «médecins \& chirurgiens» étaient spécifiquement invités à concourir, ne serait-ce qu'en envoyant des informations sur les «traitements des maladies épidémiques ou celles qui règnent dans les hôpitaux». Lenoir et Vicq d'Azyr

Hilaire-Pérez (2000); Briggs (1991).

55 Tout le dossier est conservé dans le fonds JdF 582.

56 Histoire et Mémoires de l'Académie des Sciences, 1744, p. 83.

57 Entre autres mentions, dans le plan de travail pour les inspecteurs déjà évoqué (BnF Arsenal ms 10015), tout comme dans ses Mémoires, op. cit., p. 579.

58 Journal des Sçavans (désormais $J d S$ ), p. 699.

$59 \quad J d S$, 1783, pp. 434-435.

${ }^{60} J d S$, Séance publique de la Société Royale de Médecine du 15 février 1785, pp. 310-311. 
ont-ils agi délibérément en repoussant plusieurs fois les prix proposés, en vue de recueillir une quantité importante d'informations qui permettraient, par la suite et sur le modèle des «topographies médicales », de dresser une synthèse sur ces sujets déterminés? Rien ne permet de le prouver, mais la constitution de cette Société Royale de Médecine s'est assurément effectuée à partir de l'idée d'une accumulation de connaissances qui permettrait ensuite d'agir de façon rationnelle. Le canal entre la Société Royale de Médecine et les administrations ne passait pas uniquement par la Lieutenance, mais celui-ci semble avoir été structurant.

Le successeur de Lenoir ne changea pas de méthode, en s'impliquant notamment dans le prix proposé par l'Académie Royale des Sciences en 1789 pour l'année 1790 relatif à la «meilleure manière de curer les puits \& de vuidanger les fosses d'aisance $»^{61}$, répondant à un problème majeur de salubrité et de sûreté publiques de la décennie 1780-1790. La diffusion de cette implication policière par un des plus grands journaux du royaume, le Journal des Savants, qui faisait de plus autorité dans le domaine scientifique, tendit à fixer les sujets qui méritaient d'être étudiés. La Lieutenance se servit ainsi du Journal et mobilisa les institutions scientifiques pour orienter les débats vers des sujets qui permettraient d'améliorer le service de la police et, plus généralement, l'action de l'État. Par ailleurs, elle ne manqua pas de le faire savoir, dès la mise en place d'entreprises privées répondant à une mission de police.

\section{Les soumissions et les marchés publics policiers}

Lenoir ne se contenta pas, pour répondre aux besoins de la Lieutenance, de passer par le canal des concours académiques. Il eût d'ailleurs été difficile de le faire pour améliorer le service strictement dit du nettoyage des rues ou de l'éclairage.

Sartine, en son temps, avait autorisé une société que Bourgeois de Chateaublanc, récompensé en 1763, avait initiée avec un fermier général, Tourtille Saugrain, et Pierre-Joseph Lavalar, commissaire général à la voierie. L'affaire, assez rapidement menée, aboutit à un arrêt du Conseil du roi du 30 juin 1769 qui faisait passer le service de l'éclairage d'un système municipal et policier à une entreprise privée sous le contrôle de la Lieutenance ${ }^{62}$. Les soumissionnaires de ce contrat avec la monarchie s'offrirent par le vecteur du canal académique, et par un réseau d'informations propre aux cercles administratifs et financiers parisiens. Lenoir, d'ailleurs, reconduisit le contrat au profit du seul Saugrain en $1784^{63}$, ce qui lui valut de nombreuses accusations de corruption.

Mais Lenoir dépassa ce strict cadre parisien pour canaliser les initiatives entrepreneuriales. Sa conception du «service de police» ne restreignait pas l'action policière à la seule sphère des agents de la Lieutenance et des officiers monarchiques. Il systématisa le recours à des entrepreneurs privés pour remplir certaines fonctions de police en relation étroite avec l'espace urbain. Ainsi, il contribua à attribuer à une compagnie du Ventilateur, dont faisait partie Cadet de Vaux, un privilège pour la ventilation des fosses d'aisance au moment de leur nettoyage ${ }^{64}$. On peut voir dans

\footnotetext{
$61 \quad J d S, 1789$, p. 500.

62 AN, F13 351, «Éclairage de la ville de Paris».

63 Ibid., nouvelle soumission de Saugrain à l'initiative de Lenoir, septembre 1784.

64 Sur cette question, Chagniot (1988, p. 176sq.); Le Roux (2011, p. 85sq.).
} 
ces entreprises privées agissant pour le «bien du public» une forme de pré-service public, comportant un contrat, des objectifs à atteindre et le contrôle par l'État d'une certaine forme d'action ${ }^{65}$. Pour autant, il manquait les formes de soumission. Elles existèrent dans le cas du nettoyage des rues et des marchés, et c'est ici que Lenoir se déprit des formes les plus institutionnelles d'appel au public, puisqu'il recourut en 1779 au moyen du «prospectus» pour attirer à lui des initiatives individuelles. Ce prospectus avait une double finalité: porter dans le débat public les sujets policiers et, à la suite des réponses, trouver des personnes capables d'agir selon les besoins énoncés par la Lieutenance. La publicisation des procédures administratives englobait ainsi le public dans les démarches policières, en le prenant à la fois comme témoin de l'action de la Lieutenance sur des sujets légitimes aux yeux de l'opinion publique, et en en tirant des énergies privées capables d'entreprendre la mise en œuvre de ces missions. Le système de l'adjudication, sans être une nouveauté, était ainsi renouvelé dans sa philosophie, et participait de la réélaboration permanente du lien entre monarchie et société.

Le sujet du nettoyage était en effet depuis longtemps débattu dans l'espace public, et faisait l'objet de commentaires qui pouvaient aller à l'encontre de l'image que la monarchie voulait donner de la capitale, symbole des Lumières, reflet de la splendeur de la royauté, et pourtant sale et immonde, en particulier à cause des boues. De plus, un problème pratique d'organisation du service se posait à Lenoir, sous l'effet de l'extension de l'espace bâti, qui multipliait les rues à couvrir avec des moyens constants, fixés par un système d'entrepreneur général qui sous-louait des baux à des entrepreneurs de quartier. En 1779, aucun mémoire n'emporta la conviction du Lieutenant général, mais il retint tout de même le projet de JacquesHyppolite Ronesse, qu'il fit imprimer en 1782 sous le titre Vues sur la propreté de Paris. Dans cet écrit, largement diffusé, Ronesse développait une conception englobante de l'activité policière de nettoyage des rues, qui devait tenir compte du pavé (en mauvais état), des bâtiments (qui empêchaient la circulation de l'air), des comportements sociaux (le manque d'application des règlements de police) et des infrastructures (le manque d'évacuations des eaux usagées). Il faisait ainsi écho, pleinement, aux préoccupations que l'on rencontre dans les écrits sur la ville de l'époque, tant de la part des architectes célèbres (Pierre Patte ${ }^{66}$ ) que des faiseurs de projets urbains (Poncet de La Grave ${ }^{67}$ ) ou des réformateurs policiers proches de l'Encyclopédie (Guillaute ${ }^{68}$ ). Il proposa à Ronesse une place - et non un office, moins souple - d' «Inspecteur général des préposés, et aussi des Balayeurs, des chartiers et autres manœuvres journellement employés au service du Nettoyement ${ }^{69}$. On ne sait si Ronesse accepta cette charge. Toujours est-il que Lenoir innova en matière de mobilisation des initiatives privées et élargit la sphère d'audience de la police audelà des seuls administrateurs et médecins. Sa démarche n'eut pas d'écho chez son successeur immédiat Thiroux de Crosne, mais fut réutilisé, sous d'autres conditions de transparence, sous la Révolution, lorsque l'administration municipale de Paris publia un prospectus en germinal an IV pour «l'adjudication de l'entreprise générale

\footnotetext{
Margairaz (2005).

66 Patte $(1766,1769)$.

67 Lemas (2002a).

68 Guillaute (1974).

${ }^{69}$ Milliot (2011, p. 590).
} 
du nettoiement de Paris $\gg^{70}$. La différence essentielle tient dans le fait que sous la Révolution, une véritable procédure de soumission publique fut établie et suivie, avec pas moins de sept candidats dont les soumissions furent rendues publiques par voie d'affichage. La décision était ainsi mise sous les yeux du public et justifiée, dans une exigence de transparence impliquant un lien véritable entre administrateurs et population, les premiers n'étant que les mandataires des seconds. Lenoir, lui, vivait en partie les exigences du public comme une contrainte. En adaptant et en renouvelant la pratique ancienne de l'adjudication, il contribua pourtant à accroître les exigences de contrôle de l'action policière en recourant aux initiatives privées, puisqu'il s'adressait à la population pour fournir (pallier?) un service public jugé par tous défaillant.

À ce titre, une véritable dialectique entre public et administration policière s'installa, faite de contrainte et d'appels de part et d'autre, mais dictée par la composition sociologique de ceux qui pouvaient parler et s'exprimer ${ }^{71}$, principalement $^{\text {' }}$ par le moyen de l'imprimé, et ainsi peser sur les choix et les orientations des politiques policières en matière d'espace urbain. La volonté des autorités révolutionnaires fut d'ouvrir ces questions à tous ceux qui étaient concernés, sans pour autant déstructurer un service qui devait être, compte tenu des conditions ${ }^{72}$, singulièrement amélioré, voire restauré.

En dernier lieu, Sartine et ses successeurs ont utilisé à des fins de communication certains ouvrages imprimés et organes de presse les plus renommés du moment.

\section{LA MOBILISATION DES RÉSEAUX MÉDIATIQUES}

La diffusion de l'imprimé s'accrut de manière très forte dans la seconde moitié du XVIII ${ }^{e}$ siècle. Tout en gardant à l'esprit que, dans le contexte urbain, les livres n'étaient pas les seules sources écrites et imprimées qui s'offraient aux Parisiens ${ }^{73}$, l'action de la Lieutenance dans les domaines des livres et de la presse dévoile une véritable politique de publicisation de la police.

\section{Les publications "policières"}

Dussausoy, dont l'ouvrage Le Citoyen désintéressé fut publié pour accompagner l'aménagement des nouvelles halles sur le terrain de l'hôtel de Soissons, ne constitue pas un cas isolé. Mais il ne fut pas utilisé par Sartine pour simplement propager et vanter les réformes policières dans le domaine urbanistique. Il développa de nouvelles idées et diffusa des expériences étrangères, dans le but de stimuler les initiatives dans l'espace urbain. L'auteur expliqua en effet dans sa préface que le Lieutenant général, «informé des recherches [qu'il] avoi[t] faites [au sujet des incendies], [lui] ordonna de dresser un projet ${ }^{74}$. Son expérience était le fruit de

\footnotetext{
$\overline{70 \quad \text { AN, F7 4340. }}$

71 Rancière (1995).

72 De nombreux rapports de police de l'an III, spécialement pendant l'hiver, marquèrent l'état déplorable du nettoyage des rues de Paris. AN, AF 1471.

73 Roche (1993).

74 Dussausoy (1767, p. 15).
} 
voyages à Londres et Amsterdam, deux villes présentées comme des références en matière de police et d'organisation politique, et auxquelles les écrivains français comparaient fréquemment la capitale française, la première en raison de sa taille et sa population, la seconde pour certains de ses services de sûreté urbaine. L'objectif de l'ouvrage, au-delà des projets grandioses d'aménagements de places ou de rues, visait à «perfectionner» les dispositifs de lutte contre l'incendie et de distribution des approvisionnements. Il s'insérait dans une littérature en profusion ${ }^{75}$, mais était le fruit d'une commande, voire d'un contrôle, de la part de la Lieutenance de police qui montre son insertion dans le champ des écrits réformateurs publics.

Une littérature de combat fut également l'objet de l'attention policière. Les Mémoires de Lenoir nous apprennent que Sartine, son prédécesseur, fit composer, imprimer et publier en 1767 un ouvrage intitulé L'enthousiasme françois pour répondre aux critiques publiques contre le manque de liberté ${ }^{76}$. Ces critiques reposaient, en sus des questions économiques, sur la dénonciation de l'usage des lettres de cachet, dont certains parlementaires souhaitaient réformer les abus. L'auteur de l'ouvrage de commande, Marchand, était lui-même avocat et censeur. La structuration de son propos annonce le discours de Sartine devant le Parlement du 28 novembre 1768. Des enthousiasmes excessifs étaient dénoncés, dans les domaines de la médecine (à propos de l'inoculation), de l'attrait pour les modèles politiques et intellectuels étrangers, des attaques contre les «gens en place», des spectacles ou du luxe. L'excès d'enthousiasme conduisait, de manière assez mécanique, le «peuple de Paris» à s'échauffer, se diviser et se disputer, ce qui pouvait entraîner des conséquences politiques graves, l'auteur se référant dès le début de son propos aux exemples des Armagnacs et des Bourguignons, ou à celui de la Ligue. La démonstration s'achevait par des réflexions sur la liberté, dont il ne fallait pas «abuser ${ }^{77}$. Selon l'auteur, «La vraie liberté est le droit de faire sans inquiétude tout ce qui n'est contraire ni aux Loix, ni au bien général. Ce qui est au-delà n'est qu'une licence contraire au droit divin \& à l'ordre politique». On comprend ainsi mieux la crainte des autorités policières à propos des débats publics, et de la constitution d'une opinion publique qui pût être opposée au système politique en place. Certes, la censure fut alors utilisée comme une arme pour résorber, atténuer, voire étouffer les critiques susceptibles de remettre en cause certains fondements de l'ordre social et politique ${ }^{78}$. Mais elle ne fut pas la seule. Une véritable politique policière d'insertion dans le débat public exista, qui compléta les dispositifs de censure et de contrôle en amont de la littérature politique et critique. Sartine, tout comme Lenoir mais dans des styles différents, entretinrent en effet des liens parfois cordiaux avec les "philosophes», en les recevant et discutant avec eux de leurs publications, de manière à prévenir les éventuels excès commis par ces derniers. Ainsi, la correspondance de Diderot fut

75 On peut recenser, à la même période, deux autres publications particulièrement élogieuses à l'endroit de la Lieutenance, et dont la structure reflète l'organisation policière de la ville en vingt quartiers, et pour chacun desquels le nombre de lanternes et les dispositifs policiers sont présentés: Le Sage (1769), 2 t. et Jèze (1772).

76 Anonyme [Marchand] (1766).

77 Ibid., p. 65.

78 Roche, Darnton (1989). 
très fournie avec Sartine ${ }^{79}$. En 1762, le philosophe n'hésita pas à se rendre chez le Lieutenant général de police pour lui reprocher d'avoir introduit dans sa maison un espion, ce qui fit rire Sartine ${ }^{80}$. L'acceptation, à des degrés divers, d'un même système policier de contrôle du monde philosophique de la part des contrôlés et des contrôleurs montre certaines affinités intellectuelles qui existaient entre ces deux pôles. D'ailleurs, l'ouvrage de Marchand, dont on peut prêter les mots à Sartine, affirma: «Dans quel lieu du monde cette liberté resserrée dans ses justes bornes se trouve-t-elle mieux établie que parmi nous ? $\gg^{81}$. Il faisait écho au niveau politique aux réflexions de Diderot à propos du commerce des grains, qui estimait que la «première condition d'une société» n'était pas la richesse et la liberté, mais la sûreté 82 , ce que Sartine s'employait à garantir. La confluence intellectuelle entre certains philosophes et les administrateurs de la police, trouva un débouché dans les moyens employés pour contrôler et participer à la sphère publique des débats politiques et économiques. Il eût été vain, sans doute, de tenter d'interdire toute publication un tant soit peu critique. La Lieutenance, au fait des outils et des armes de son temps, s'inséra donc dans l'espace public en faisant publier, plus ou moins directement, des ouvrages tendant à orienter les débats et à créer une sphère de dialogue consensuel qui ne remît pas en cause les fondements du pouvoir. Cette gouvernementalité à la fois souple et autoritaire ne permit pas d'éteindre toutes les critiques, et les pratiques répressives restèrent largement de mise, attisant, selon Lenoir, les ressentiments des publicistes contre la police ${ }^{83}$. Ce ressentiment éclata au grand jour au début de la Révolution, et visa spécialement Sartine et Lenoir, dans une campagne publique de dénigrement, à laquelle l'ancien Lieutenant général de police souhaitait répondre ${ }^{84}$. On comprend ainsi que, même au-delà de la césure de 1789, la dialectique entre police et public critique se poursuivit et entraîna la rédaction d'ouvrages qui, bien qu'évoquant l'Ancien Régime, tendaient à mettre sous les feux des réflexions la situation présente. Ce lien avec le présent existait dès avant 1789 , notamment quand la Lieutenance générale s'intéressa de près à la presse de grande diffusion, et s'ingénia à contrôler certaines publications ou à s'insérer dans la relation des événements quotidiens.

\section{Les liens avec la presse}

Tout comme pour les livres et leur diffusion démultipliée, la deuxième moitié du XVIII ${ }^{e}$ siècle connut une croissance formidable des organes de presse et une extension de l'espace qu'ils couvraient. Deux des principaux journaux étaient le Journal de Savants, fondé en 1665, et qui supplanta peut-être le Mercure français ${ }^{85}$, et le Journal de Paris, dont le premier numéro parut le $1^{\text {er }}$ janvier 1777. Un de ses fondateurs était Cadet le Jeune, frère de Cadet de Vaux, tous deux très liés à Lenoir et qui firent du journal, selon Nicole Brondel, «l'organe de diffusion des réformes

\footnotetext{
Versini (1997).

80 Ibid., lettre à Sophie Volland du 19 septembre 1762, pp. 440-441.

81 Marchand (1766, p. 64).

82 Versini (1997), t. III, Politique, «Apologie de l'abbé Galiani», p. 133.

83 Milliot (2011, p. 1008sq., «Liberté de presse, opinion publique»).

84 Milliot (2011 «Défendre et construire la police» et 2013).

85 Vittu (1991).
} 
gouvernementales, la propagande des Lumières ${ }^{86}$. Cette idée de propagande doit être nuancée, car le Journal sut faire preuve d'esprit critique qui lui valut les foudres de la censure et de la police elle-même ${ }^{87}$. Il fut malgré tout un organe de diffusion de la parole policière, ainsi qu'un moyen d'exposition pédagogique de l'action de la Lieutenance.

Lenoir n'hésita pas à imposer dans le Journal des Savants la mention de certains ouvrages qui intéressaient directement la police urbaine, notamment dans ses liens renforcés avec le monde scientifique. En 1774, J.-P. Macquer fut nommé directeur du mensuel scientifique de référence. Le 20 septembre 1774, le Lieutenant général lui écrivit pour lui demander de faire mention dans le Journal de l'ouvrage du médecin ordinaire du roi Raulin sur les eaux minérales ${ }^{88}$. Un grand débat, tant intellectuel que politique, avait alors lieu à propos de la qualité des eaux en France, et spécialement celles de Paris (la Seine au premier rang). Le médecin fut alors nommé «par le Gouvernement» pour analyser la composition et évaluer les qualités respectives des eaux minérales. Macquer accorda à l'ouvrage un «extrait», c'est-à-dire un compte rendu de cinq pages, ce qui n'est pas négligeable. Après une présentation sommaire de chacune des idées contenues dans les quinze chapitres de l'ouvrage, il ne manqua pas de préciser en citant le livre lui-même que «les nombreuses matières [...] auroient exigé un Volume considérable [...]. Mais le Ministère, qui en désire la publication, a donné ordre de le réduire en un précis succinct » qui visait à «faire connoître les eaux minérales, leur vertus, les maladies auxquelles elles sont propres, \& les moyens d'en faire usage ${ }^{89}$. Le compte rendu se poursuivit alors par trois pages de commentaires désabusés et parfois narquois sur le manque de connaissances criant de Raulin dans le domaine de la Chimie, qui invalidait la plupart de ses propositions, empruntées à une vieille philosophie des éléments. Macquer conclut ainsi l'article:

Nous ne pousserons pas plus loin ces observations relatives à la Chimie; M. Raulin, livré à des occupations de pratique médicinale, totalement étrangères à la Chimie, est très-excusable sans doute, de n'être point versé dans cette Science, comme ceux qui en font leur principal objet; mais elle prouvent qu'il n'est pas possible de traiter avec succès de la nature des principes des eaux minérales, sans être fort savant en Chimie ${ }^{90}$.

Derrière la critique virulente et purement scientifique, le Journal des Savants concourait toutefois à la recherche de «l'utilité» propre aux administrations, en particulier celle de la police, partagée avec les «philosophes» du siècle des Lumières. La dénonciation de la méthode de Raulin participait d'une reconfiguration des sciences du temps, dans laquelle la chimie, menée par des figures fortes, occupait une place prépondérante et en expansion. Elle rompait en partie avec les pratiques traditionnelles de la science classique, dont Raulin était un bon représentant. De plus, Raulin incarnait, aux yeux de Lenoir, un blocage administratif, dans la mesure

\footnotetext{
$\overline{86}$ Brondel (1991). L'auteure reprend l'idée habermasienne d'un journal destiné à un public bourgeois pour qui les affaires urbaines sont un marchepied vers la politique.

87 Ainsi Beaumarchais fut-il emprisonné à Saint-Lazare après avoir fait paraître un mot sur la Reine dans ce Journal de Paris, ce dont Lenoir rend compte dans ses Mémoires, op. cit., p. 1013.

$88 \mathrm{BnF}, \mathrm{ms}$ fr. 12305 , fol. $508 \mathrm{r}^{\circ}$.

$89 \quad J d S$, septembre 1774, pp. 611-616.

$90 \quad$ Ibid., p. 616 .
} 
où il s'opposait à une réforme de la Commission établie en 1772 pour évaluer les remèdes et les eaux minérales. La mise au pilori scientifique établie par le Journal des Savants fut donc une arme pour Lenoir pour justifier de l'inutilité de cette Commission et préparer le transfert de ses attributions à la future Société Royale de Médecine ${ }^{91}$.

La teneur du compte rendu ne valut pas de problèmes à Macquer. Il reçut en effet du successeur temporaire de Lenoir, Albert (le bras droit de Turgot) une lettre pour lui demander son avis sur des mémoires relatifs aux ustensiles de cuisine et à l'usage du cuivre dans leur fabrication ${ }^{92}$. Le recours à cette autorité (il était membre de la Faculté de médecine) montre que la Lieutenance créa un système combinant la sphère scientifique, l'administration et la publication des résultats de cette entente en vue de "prévenir les accidents ${ }^{93}$ et de procurer au public la plus grande utilité.

Le Journal des Savants, par la suite, publia les concours proposés par les Lieutenants généraux, en rapport bien souvent avec la santé publique. Il rendit compte fréquemment d'ouvrages qui intéressaient la police, en particulier ceux de la santé, en relatant les étapes de la lutte des autorités parisiennes (municipalité et Lieutenance) en faveur des personnes noyées, sous la direction de M. Pia ${ }^{94}$, ou encore les ouvrages relatifs à la petite vérole ${ }^{95}$, à laquelle Lenoir s'intéressait particulièrement, ou à propos des recherches menées pour diminuer les risques liés aux gaz méphitiques des fosses d'aisance ${ }^{96}$ ou des cimetières ${ }^{97}$. Pour ce dernier sujet, le Journal des Savants rendit compte de mémoires d'académiciens ou d'ouvrages médicaux d'importance, en particulier pour le cimetière des Innocents à Paris, qui mobilisa la Lieutenance et le Parlement pendant de longues années afin d'expulser ce lieu «dangereux » de l'intérieur de la ville. La Lieutenance parvint finalement à exhumer les corps grâce au travail de Legrand et Molinos, ses deux architectes en $1785-1786^{98}$, ce qui donna lieu à un rapport de Thouret, de la Société Royale de Médecine, chargé de surveiller les opérations. La publication de ce rapport entraîna une émulation scientifique entretenue par le Journal dont l'un des collaborateurs, l'abbé Tessier, fit part de ses expériences sur le sujet à Rambouillet. Le Journal des Savants conclut en 1791 que ces travaux étaient nécessaires pour «l'utilité publique $\&$ le progrès de la science $»^{99}$, ce qui perpétua les liens systémiques entre la sphère administrative policière, les institutions scientifiques et la presse.

Sans être inféodé aux desiderata de la Lieutenance, le Journal des Savants, qui ne traitait pas les sujets économiques, contribua à mettre en question la police et ses pratiques, notamment par ses extraits d'ouvrage juridiques ou historiques, les deux étant alors très liés. Une question parcourut le Journal tout au long des années 1770-1790, qui fut celle de la réforme des lois, tant civiles que pénales. Des projets, souvent ambitieux, furent présentés. Tous insistèrent plus ou moins

\footnotetext{
$91 \quad \mathrm{BnF}, \mathrm{JdF} 499$.

$92 \mathrm{BnF}, \mathrm{ms}$ fr 12305 , lettre du 27 juillet 1775 , fol. $10 \mathrm{r}^{\circ}$.

93 Ibid.

$94 \quad J d S, 1774$, p. $812 ; 1776$, p. 23 , etc.

$95 J d S, 1777$, p. $289 ; 1786$, p. 378 , etc.

$96 J d S, 1778$, p. $845 ; 1779$, p. $45 ; 1782$, p. 4351785 , p. 50, etc.

$97 J d S, 1763$, p. $232 ; 1789$, p. $435 ; 1791$, p. 2.

98 Nous nous permettons de renvoyer à notre travail, Vidoni (2011, pp. 302-304).

99

$J d S, 1791$, p. 8.
} 
sur le nécessaire respect de procédures fixées pour éviter l'arbitraire. La plupart de ces ouvrages évoquaient la police, entendue comme une part de l'activité souveraine de régulation des désordres, et à ce titre toujours en partie intégrée à la justice. Cette floraison d'écrits réformateurs, sans remettre en cause directement la Lieutenance, tendirent à placer au centre des débats les pratiques de police. À côté des questions de «police médicale» et de police urbanistique, l'histoire et le droit contribuèrent à faire de la police au sens large un objet d'appréciation et de réflexion pour le public éclairé.

Le recentrage de la police comme thème de débat dans l'espace public imposa à Lenoir de réagir plus immédiatement et d'adapter son dispositif de communication à une opinion friande de nouveautés et d'informations sur la ville. Pour cela, il investit un nouveau média à succès, le Journal de Paris. Une rubrique «Police» fut insérée dans le journal quotidien dès le numéro 4. Elle n'avait pas pour objectif, comme la rubrique «Administration», de seulement indiquer les arrêts du Conseil ou les règlements de police les plus importants. Elle visait à fournir des informations pratiques, et à mettre en relation le public parisien potentiel et l'administration policière. Ainsi, le 8 janvier 1777, un article informait, à propos de la salubrité des rues (et après avoir rappelé le paradigme policier qui voulait qu'elle contribuât à la «salubrité de l'air \& à la commodité du Public») que des balayeurs employés par les entrepreneurs du nettoyage pouvaient être rémunérés (modiquement) pour balayer le devant des maisons des particuliers. Les commissaires du Châtelet étaient chargés de mettre en relation les demandeurs et les offrants, ce qui aurait également pour effet de diminuer le chômage des manouvriers pendant la saison hivernale. L'utilité pratique de la rubrique «Police» fut enrichie par une publicité pour le Bureau des ramoneurs, qui proposait aux habitants des particuliers pour pallier les défauts des ramoneurs privés et domestiques ${ }^{100}$. Ils étaient enregistrés par la Lieutenance sous un numéro, et donc identifiables et contrôlables, de même qu'habillés « uniformément » et tenus de porter secours en cas d'incendie, fléau qu'ils avaient la tâche de prévenir. L'action préventive de la Lieutenance était d'ailleurs largement explicitée dans cette rubrique, quitte parfois à imbriquer plusieurs aspects du travail policier, notamment quand des améliorations techniques portées aux tombereaux chargés d'enlever les boues étaient présentées en détail ${ }^{101}$. Elles permettaient de protéger les habitants des désagréments des projections, mais encore de prévenir les accidents de la circulation par le renforcement des essieux et ainsi assurer la vie des passants et des piétons ${ }^{102}$. De la sorte, les rues de la capitale étaient plus sûres, plus belles et plus propres, ce qui permettait d'augmenter la grandeur de Paris. Loin de ne constituer qu'une simple information technique, la rubrique «Police» permettait à différents publics, des scientifiques curieux aux faiseurs de projets, de s'intéresser à l'action de la Lieutenance et aux succès de l'administration pour maîtriser un espace urbain encore perçu comme confus, encombré et dangereux ${ }^{103}$. Le Journal de Paris était également le moyen de diffuser un appel aux initiatives particulières, complétant ainsi les annonces officielles des concours, des prix académiques et des prospectus. Dans le $\mathrm{n}^{\circ} 320$, Lenoir fit appel aux «Artistes » pour améliorer les fameux «essieux »

\footnotetext{
$100 \quad$ Journal de Paris (désormais $J d P$ ), 19 juillet 1777, n 200 , pp. 3-4.

$101 J d P, 6$ mars $1777, \mathrm{n}^{\circ} 65$, p. 2.

$102 J d P, 16$ novembre $1777, \mathrm{n}^{\circ} 320$, p. 3.

103 Pour quelques pages sur ces désordres urbains, Chagniot (1988, chap. VI).
} 
des tombereaux, et promettait d'en faire l'essai publiquement, renforçant le rôle de la Lieutenance dans le processus de sélection économique et d'innovation ${ }^{104}$.

À côté de cette rubrique «Police», une autre rubrique fut largement consacrée aux affaires de police, mais sous un angle différent. Il s'agissait de la rubrique «Événement». Elle n'était pas pourvue exclusivement d'informations policières, comme la précédente. Elle visait à relater, sur le ton du récit parfois anodin, des situations exceptionnelles ou extraordinaires qui pouvaient survenir partout en France, mais de préférence à Paris. Ainsi, le numéro 42 (11 février 1777) contenait le récit du sauvetage de quatre nettoyeurs de fosses d'aisances et la mort de deux de leurs compagnons à Toulouse, sous l'effet des gaz méphitiques. L'aspect sensationnel du récit, ainsi que la tragédie rapportée, ne pouvaient qu'inviter le public à réfléchir sur la dangerosité des fosses d'aisance. Ce thème fut fréquemment évoqué, et trouva une issue heureuse en 1781, quand le Journal se fit l'écho d'une expérience menée le 16 juin par Cadet de Vaux pour assainir «l'égout de la Porte St Antoine», où plusieurs personnes étaient décédées quelques jours plus tôt ${ }^{105}$. L'emploi du «ventilateur» développé par le même, ainsi que le fruit des recherches de la Société Royale de Médecine sur des «acides» utiles pour rétablir les personnes suffoquées, montrent l'intrication entre entreprise privée chargée d'un service au public, presse quotidienne parisienne et Lieutenance générale de police. La rubrique «Événement» contenait d'ailleurs très fréquemment le récit d'incendies survenus dans la ville, et auxquels la police, ainsi que le corps des pompiers (sous les ordres du Lieutenant, et qui avait été réorganisé par Sartine sur le modèle militaire), parvenait à remédier grâce aux innovations techniques et aux nouveaux dispositifs d'occupation de l'espace parisien. Les cérémonies policières de célébration des pompiers morts pendant leur service ${ }^{106}$ furent d'ailleurs relayées par le Journal de Paris, en particulier au moment de l'incendie de l'Opéra, haut lieu pour le public parisien ${ }^{107}$, qui fit naître une controverse à propos des lieux dangereux dans la ville à laquelle la Lieutenance prit toute sa part.

Enfin, une troisième rubrique, apparue en 1782, intitulée «Établissement», vanta haut et fort les réalisations policières institutionnelles, en débutant par le récit de la fondation de l'hospice de Vaugirard pour les nouveau-nés atteints de petite vérole, dont Lenoir fut à l'initiative.

La dernière particularité relative au Journal de Paris fut un espace de dialogue ouvert au cours de l'année 1777, dans laquelle un débat fut initié à propos des aménagements urbains nécessaires pour la capitale, et dans lesquels la police devait jouer un rôle de première importance. Une lettre faussement anonyme fut publiée par le Journal le 4 juin 1777, dans laquelle son auteur affirmait vouloir «traiter [...] par des Lettres courtes \& détachées, les grands objets qui intéressent le monde, comme, par exemple, plusieurs points de Police \& d'Administration relativement à la sûreté $\&$ à la commodité des Citoyens, aux bonnes mœurs \& à l'ordre public [...]». Le but de celui qui allait devenir un correspondant assidu du journal était de «provoqu[er]» les critiques et les discussions dans cet espace public qu'était devenu le Journal de Paris. Il signa sa deuxième lettre du 12 juin 1777 du nom de «Pro Patria», en

\footnotetext{
104 Ibid.

$105 J d P, 25$ juillet $1781, \mathrm{n}^{\circ} 206$.

$106 J d P, 3$ septembre $1781, \mathrm{n}^{\circ} 246$.

107 Chagniot (1988, p. 376sq.).
} 
insistant cette fois sur le nécessaire élargissement des rues pour permettre un passage plus aisé et plus sûr des voitures qui permettrait de diminuer les risques pour les piétons. Le second avantage des rues larges était la meilleure circulation de l'air, que l'on devait retrouver dans les hôpitaux qu'il souhaitait voir éloignés de la ville. Quatre lettres de Pro Patria suivirent, roulant sur le thème de la création d'un Conseil composé d'architectes pour tracer un «plan de réformation dans les constructions qui forment la Ville de Paris ${ }^{108}$ qui serait soumis aux commentaires des citoyens en étant affiché dans l'Hôtel de Ville. L'usage de l'outil cartographique, commun à nombre d'administrations et spécialement la Lieutenance, permettrait une meilleure diffusion des projets urbanistiques et à la critique publique de s'exprimer. Les autres lettres complétèrent ce projet, en prévoyant notamment que la puissance publique récupèrerait les terrains abandonnés par les propriétaires pour bâtir et aménager des carrefours, des fontaines ${ }^{109}$ ou d'autres ouvrages d'utilité publique tels que les quais et les places, dont il fallait absolument détruire les bâtiments ${ }^{110}$. La dernière lettre, publiée quelques mois plus tard, défendit le style lent de Pro Patria, qui justifiait l'interruption de sa correspondance par le fait qu'aucun «Plan d'embellissement de Paris» n'avait été arrêté. L'objectif de cet écrivain était donc bel et bien de susciter une action réformatrice de la part des autorités, ce qui échoua rapidement et justifia l'arrêt de cette correspondance. En revanche, elle entraîna de nombreuses réactions, puisqu'au cours de ces six mois de correspondance, pas moins de cinq lettres attaquant l'auteur furent publiées. La première émanait d'un soi-disant négociant d'Amsterdam, qui signait «Nieman» (cet inconnu fut un correspondant prolixe pour le Journal par la suite) et qui affirmait que Pro Patria se trompait de priorité. Il était absolument nécessaire, pour assainir la ville, de nettoyer avant tout l'intérieur des maisons, dont la saleté révoltait «les sens» et annonçait «les dangers dont on est entouré: l'air infecté qu'on respire, les alimens dissous avec le poison dans leur préparation, le contact malpropre de tous les ustensiles qu'on emploie à son usage ${ }^{111}$. Partageant les mêmes impératifs de salubrité publique, les projets divergeaient dans les moyens employés. Nieman, d'ailleurs, continua de mener une campagne en faveur de l'assainissement des intérieurs, en demandant une meilleure distribution de l'eau et en soutenant, sans les nommer, les projets des frères Perier ${ }^{12}$. Il poursuivit par quelques lettres qui évoquaient le problème des nourrices, que Lenoir s'employait à réguler et contrôler afin de diminuer la mortalité infantile ${ }^{113}$.

Les deux critiques suivantes concernèrent un père qui craignait de reconnaître son fils dans les lettres de Pro Patria ${ }^{114}$, craintes qui furent démenties le lendemain par le fils. À l'occasion, le projet était décrié par le père, mais défendu par le fils, qui estimait que Pro Patria employait un style «grave et utile». Il ajoutait: «Les gens à projets, les dissertateurs en alignemens, les commentateurs du Commissaire La

\footnotetext{
$108 J d P, 20$ juin $1777, \mathrm{n}^{\circ} 171$.

$109 J d P, 1^{\text {er }}$ juillet $1777, \mathrm{n}^{\circ} 182$.

$110 J d P, 20$ juillet $1777, \mathrm{n}^{\circ} 201$

$111 J d P, 26$ juin $1777, \mathrm{n}^{\circ} 177$.

$112 J d P, 30$ juillet $1777, \mathrm{n}^{\circ} 212$, dans lequel il dénonce la fainéantise des domestiques qui ne nettoient pas les intérieurs, ce qui correspondait à la vision que Lenoir avait de ce groupe considéré comme dangereux et indolent par nature.

$113 J d P, 4$ août 1777, $\mathrm{n}^{\circ}$ 216. Sur les nourrices, Lenoir, Détail de quelques établissements, op. cit.

$114 J d P, 25$ juillet 1777, $\mathrm{n}^{\circ}$ 206, lettre de M. Mignonnet, ancien consul et quartenier de Paris.
} 
Mare embrassent [ce style], M. Pro Patria le traite avec distinction». La quatrième lettre fut le fruit des réflexions d'un certain Braden. Il attaqua avec violence le projet de Pro Patria, le comparant à un «vieux jeu de cartes» agencées «dans un vaste grenier» pour former le «modèle en relief d'un nouveau Paris». Un groupe de chats, passés par les fenêtres, mettaient à bas ce projet. Dans un style ironique et mordant, Braden écrivait: «Le désespoir s'empara de son âme, \& comme un nouvel Énée, il ne voulut point quitter ses ruines sans avoir assommé quelques Grecs. On prétend que cet accident a dérangé les organes de son cerveau, \& qu'il ne s'occupe plus qu'à écrire ses projets ». La charge, violente, détruisait le propos de Pro Patria, dont ne vint aucune réponse, si ce n'est la dernière lettre évoquée justifiant son style face à une ultime critique, celle du «Marin» (autre personnage anonyme écrivant fréquemment au Journal) parue dans le numéro du 12 décembre $1777^{115}$.

L'espace de débat s'était transformé en espace de critique acerbe et acérée face à l'impréparation et à la généralité du propos de Pro Patria, dont la correspondance cessa alors. Qui se cachait derrière ces pseudonymes? Nous ne pouvons le savoir en l'état de nos recherches. Mais Pro Patria pouvait être un agent de l'administration chargé de justifier et d'initier des projets urbanistiques dans un sens policier. Il parvint à susciter un débat et à créer un front uni et soutenu de critiques virulentes à son endroit, qui mériterait d'être mieux connu si les archives du Journal pouvaient être croisées avec des archives privées. Ce mur d'hostilité explique la fin de cette correspondance, et montre que la police et l'administration, en particulier lorsqu'elles étaient corrélées aux questions urbanistiques, soulevaient dans le «public» des réflexions qui pouvaient dépasser le strict respect des lois et de l'organisation politique et religieuse du royaume que Pro Patria s'était fixé ${ }^{116}$.

La police, entendue comme une pratique, devint l'objet des réflexions et des critiques positives et négatives de la part du public dans la deuxième moitié du XVIII ${ }^{e}$ siècle. Mise sous les lumières de cette critique dans les domaines de l'urbanisme, du droit et de l'histoire, elle appela une réaction de la part de la Lieutenance générale de police, l'institution la plus puissante qui en était chargée. Cette Lieutenance publicisa son action et sa volonté d'améliorer le service du public en diffusant dans la presse et par des ouvrages importants ses hauts faits et son action quotidienne. Ces deux mouvements se rencontrèrent pour placer au cœur du débat public, et donc de «l'espace public», une activité qui, sur le terrain de l'espace urbain parisien, s'offrait à la vue de tous et était présentée par ailleurs comme un modèle de rationalité et d'efficacité administrative. La conjonction de ces deux mouvements plaça l'opinion publique dans la position d'apprécier l'action de la Lieutenance, ce que, paradoxalement, Sartine et plus encore Lenoir souhaitaient éviter tout en recourant eux aussi à l'opinion publique pour justifier leur politique policière. La police était donc loin d'être une activité strictement secrète, bien que tout ne fût pas dévoilé. La Lieutenance mena une politique de publicité policière partielle, du terrain quotidien des rues aux hautes sphères de l'imprimé. Elle répondait à une attente, et la devançait pour certains sujets. Elle contribua donc à faire de la «police» un sujet de débat autour des questions urbanistiques. La Lieutenance fut ainsi prise dans les processus complexes d'évolution des polices des Lumières, qui se voulaient pour certaines

\footnotetext{
$115 J d P, 27$ décembre $1777, \mathrm{n}^{\circ} 361$.

$116 J d P, 20$ juin $1777, \mathrm{n}^{\circ} 171$.
} 
amélioratrices ${ }^{117}$ et tendaient globalement à se réformer, selon des critères proprement policiers, mais qui découlaient et induisaient la redéfinition des rapports entre État et société, que ce soit à Bruxelles, Madrid, Naples ou Londres ${ }^{118}$. Une opinion publique européenne était d'ailleurs attentive aux questions policières, ce qui est une autre dimension du sujet. À Paris, la Lieutenance répondait aux attentes du public en l'interpellant, tout en refusant que la police et son institution soient le sujet de débats publics. Cette contradiction, à l'image du rapport de l'État au public, perdura, et posa la question de l'action administrative et de la liberté. La concomitance avec la réduction de la censure explique comment, dans les années 1788-1789, les mises en cause de la police purent s'exprimer assez facilement, notamment à partir des émeutes urbaines qui se déroulèrent contre la garde en août $1788^{119}$. Certes, la crise parlementaire affaiblissait la puissance répressive de la monarchie, mais la police se trouva contestée, sur le terrain, car elle n'était plus ce monument qui effrayait la population parisienne. On sait en effet que les informations et les nouvelles se diffusaient très vite dans toutes les catégories sociales de la ville ${ }^{120}$. Une certaine désacralisation de l'institution, liée à une intolérance croissante à l'endroit de son action quotidienne de contrôle des comportements dans l'espace urbain ${ }^{121}$, tendirent à délégitimer l'action des agents sur le terrain, en particulier la Garde et les inspecteurs, et permit la parution d'ouvrages de dénonciation du système policier parisien ${ }^{122}$, ainsi que des émeutes que la Lieutenance n'avait pas prévues ou su prévoir. Le moment révolutionnaire des années 1789-1794 se nourrit de ces débats et de cette expérience pour repenser fondamentalement l'action policière, d'un point de vue juridique et pratique, et proposer de nouvelles formes d'encadrement social placées sous le regard des citoyens qui pouvaient y prendre part, à côté des professionnels de la police.

\author{
Nicolas Vidoni \\ Les Terrasses du Parc \\ 1 , rue Champsaur \\ 13090 Aix-en-Provence \\ nicolas.vidoni@yahoo.fr
}

\title{
BIBLIOGRAPHIE
}

Anonyme [Marchand], L'Enthousiasme françois, sl [Paris], [Hérissant], 1766.

Baker, K. M., The French Revolution and the creation of modern political culture, OxfordNew York, Pergamon Press, 1987.

\footnotetext{
$\overline{117}$ Milliot (2005b, p. 41).

118 Denys (2013); Marin (2011); Emsley (2007).

119 BnF, JdF 1103, «Émeutes de Paris, 1788-1789».

120 Bastien (2006).

121 À la fin de l'Ancien Régime, les condamnations les plus nombreuses «à la police» sont prononcées pour des défauts de balayage des portes et le non-respect des horaires de fermeture des lieux de sociabilité, dont la Garde de Paris et les inspecteurs, qui agissent notamment la nuit, sont largement à l'origine. Vidoni (2011, $3^{\mathrm{e}}$ partie).

122 Lettre de Desbrugnières, inspecteur de police, à son confrère D'Agoult, Capitaine aux Gardes Françoises, Paris, ce 15 mai 1788, Paris, sn, 1788; Louis-Sébastien Mercier, dans son Tableau de Paris, n'était pas plus élogieux sur le personnel policier. Mercier (1782, rééd. 1979), «Commissaires», «Espions», «Garde de Paris».
} 
Bastien, P., L'exécution publique à Paris au XVIII siècle. Une histoire des rituels judiciaires, Paris, Champ Vallon, 2006.

Beaudeau, abbé, Avis aux honnêtes gens qui veulent bien faire, Amsterdam, 1768.

Berlière, J.-M., Policer Paris au siècle des Lumières. Les commissaires du quartier du Louvre dans la seconde moitié du XVIII e siècle, Paris, Mémoires et documents de l'École des Chartes, 91, 2012.

Boucheron, P., Offenstadt, N. (dir.), L'espace public au Moyen Âge. Débats autour de Jürgen Habermas, Paris, PUF, 2011.

Bourdelais, P. (dir.), Les hygiénistes. Enjeux, modèles et pratiques (XVIII ${ }^{e}-X X^{e}$ siècles), Paris, Belin, 2001.

Brian, E., La mesure de l'État, administrateurs et géomètres au XVIII siècle, Paris, Albin Michel, 1997.

Briggs, R., The Académie Royale des Sciences and the pursuit of utility, Past and Present, 1991, 131, pp. 38-88.

Brondel, N., Journal de Paris, in Sgard, J. (dir.), Dictionnaire des Journaux, 1600-1789, Paris, Universitas, 1991, pp. 615-627.

Brouillet, P., La maréchaussée idéale: les Essais historiques et critiques sur la maréchaussée de Cordier de Perney, 1788, in Milliot, V. (dir.), Les Mémoires policiers, 1750-1850, Écritures et pratiques policières du Siècle des Lumières au Second Empire, Rennes, PUR, 2005, pp. 169-190.

Cardona, J. L., Prendre part au siècle des Lumières. Le concours académique et la culture intellectuelle au XVIII ${ }^{\mathrm{e}}$ siècle, Annales, Histoire, Sciences sociales, 64-3, mai-juin 2009, pp. 633-662.

Chagniot, J., Nouvelle Histoire de Paris. Paris au XVIII ${ }^{e}$ siècle, Paris, Hachette, 1988.

Couture, R., Inspirer la crainte, le respect et l'amour du public: les inspecteurs de police parisiens, 1740-1789, Thèse de 1'Université du Québec à Montréal, Québec (Canada), sous la direction de Pascal Bastien et Vincent Milliot, 2013.

Denys, C., La police de Bruxelles entre réformes et révolutions (1748-1814). Police urbaine et modernité, Brepols, 2013.

Denys, C., Marin, B., Milliot, V. (dir.), Réformer la police. Les mémoires policiers en Europe au XVIII ${ }^{e}$ siècle, Rennes, PUR, 2009.

Dussausoy, M., Le Citoyen désintéressé, ou diverses idées patriotiques, concernant quelques établissements et embellissemens utiles a la ville de Paris, \& qui peuvent s'adapter aux autres Villes du Royaume, Paris, Gueffier, 1767, 2 t. en 1 vol.

Emsley, C., Crime, police and penal policy. European experiences, 1750-1940, Oxford-New York, Oxford University Press, 2007.

Farge, A., La vie fragile. Violence, pouvoirs et solidarités à Paris au XVIII siècle, Paris, Hachette, 1986.

Farge, A., Revel, J., Logiques de la foule. L'affaire des enlèvements d'enfants. Paris 1750, Paris, Hachette, 1988.

Fogel, M., Les cérémonies de l'information dans la France du XVI au XVIII ${ }^{e}$ siècle, Paris, Fayard, 1989.

Grenier, J.-Y., Histoire de la pensée politique et économique de la France d'Ancien Régime, Paris, Hachette, 2007.

Guillaute, Mémoire pour la réformation de la police de France, Soumis au roi par M. Guillaute officier de Maréchaussée de l'île-de-France, illustré de 28 dessins de Gabriel de SaintAubin, Paris, Hermann, 1974.

Habermas, J., L'espace public. Archéologie de la publicité comme dimension constitutive de la société bourgeoise, Paris, Payot, 1978. 
Harouel, J.-L., L'embellissement des villes. L'urbanisme français au XVIII ${ }^{e}$ siècle, Paris, Picard, 1993.

Hilaire-Pérez, L., L'invention technique au siècle des Lumières, Paris, Albin Michel, 2000.

Jèze, Tableau universel et raisonné de la Ville de Paris, Paris, J. P. Costard, 1772.

Jobard, F., Bavures policières? La force publique et ses usages, Paris, La Découverte, 2002.

Kaplan, S.-L., Les ventres de Paris. Pouvoir et approvisionnement dans la France d'Ancien Régime, Paris, Fayard, 1988.

Kaplan, St-L., Milliot, V., La police de Paris, une «révolution permanente »? Du commissaire Lemaire au Lieutenant de police Lenoir, les tribulations du Mémoire sur l'administration de la police (1770-1792), in Denys, C., Marin, B., Milliot, V., Réformer la police. Les mémoires policiers en Europe au XVIII' siècle, Rennes, PUR, 2009, pp. 69-115.

Lazzeri, C., Reynié, D. (dir.), La raison d'État : politique et rationalité, Paris, PUF, 1992.

Le Sage, Le Géographe Parisien, ou le Conducteur chronologique et historique des rues de Paris, Paris, Valleyre, 1769, 2 t.

Lemaire, J.-B.-Ch., La police de Paris en 1770. Mémoire inédit composé par ordre de G. de Sartine sur la demande de Marie-Thérèse. Introduction et notes de A. Gazier, Mémoires de la Société d'histoire de Paris, tome V, Paris, Champion, 1879.

Lemas, N., Le temps des Projets. Poncet de La Grave, Delamair ou l'impensé de l'urbanisme au siècle des Lumières, Histoire urbaine, juin 2002a, 5, pp. 43-66.

Lemas, N., «La ville offerte: les projets d'embellissement du XVIII siècle. Une littérature de l'accueil», Annales de la Recherche urbaine, déc. 2002b, 94, pp. 87-96.

Lenoir, J.-Ch.-P., Détail sur quelques établissemens de la Ville de Paris, demandé par Sa Majesté Impériale la Reine de Hongrie, à M. Le Noir, Conseiller d'État, LieutenantGénéral de Police, Paris, 1780.

Le Roux, T., Le Laboratoire des pollutions industrielles. Paris, 1770-1830, Paris, Albin Michel, 2011.

Mannoni, S., Une et indivisible. Storia dell'accentramento amministrativo in Francia, I. La formazione del sistema (1161-1815), Milan, Giuffrè Editore, 1994.

Manuel, P., La police de Paris dévoilée par l'un de ses administrateurs de 1789, Paris, J.-B. Garnery, L'an second de la liberté, 2 t., 1792

Margairaz, D., L'invention du «service public»: entre «changement matériel» et «contrainte de nommer », Revue d'Histoire Moderne et Contemporaine, 52-3, juillet-septembre 2005 , 52-3, pp. 10-33.

Marin, B., La réforme de police en Espagne (1768-1769): nouveaux agents et nouvelles territorialités. L'institution des alcaldes de barrio, in Borda d'Agua, F. (dir.), Police et ordre public. Vers une ville des Lumières, Condeixa-a-Nova, La ligne d'ombre, 2011, pp. 13-34.

Mercier, L.-S., Tableau de Paris, 1782, rééd. 1979, Genève, Slaktine Reprints, 12 t., 6 vol.

Meyssonnier, S., La Balance et l'Horloge. La genèse de la pensée libérale en France au XVIII siècle, Montreuil, Éd. de la Passion, 1989.

Milliot, V. (dir.), Les Mémoires policiers, 1750-1850, Écritures et pratiques policières du Siècle des Lumières au Second Empire, Rennes, PUR, 2005a.

Milliot, V., Une ville malade de son espace? Paris et le Lieutenant Général de Police Lenoir (1775-1785), in Mareck, Y. (dir.), Villes en crise? Les politiques municipales face aux pathologies urbaines (fin XVIII ${ }^{e}$-fin XX $X^{e}$ siècle), Paris, Creaphis, 2005b, pp. 32-41.

Milliot, V., Un Policier des Lumières, suivi de Mémoires de J.C.P. Lenoir, Paris, Champ Vallon, 2011.

Milliot, V., L'écriture du chaos. Les «mémoires » de Jean-Charles-Pierre Lenoir (1732-1807) ou le monde perdu d'un ancien lieutenant général de police, Annales historiques de la Révolution française, juillet-septembre 2013, 373, pp. 35-57. 
Moreau de Bellaing, C., Violences illégitimes et publicité de l'action policière, Politix, 20033, 87, pp. 119-141.

Napoli, P., Naissance de la police moderne. Pouvoir, normes, société, Paris, La Découverte, 2003.

Offenstadt, N., En place publique. Jean de Gascogne, crieur au XV siècle, Paris, Stock, 2013.

Patte, P., De la manière la plus avantageuse d'éclairer les rues d'une ville, pendant la nuit, en combinant ensemble la clarté, l'économie et la facilité du service. Par M. Patte, Architecte de S. A. S. Monseigneur le Prince Palatin, Duc régnant des Deux-Ponts, Amsterdam, 1766.

Patte, P., Mémoires sur les objets les plus importants de l'architecture, Par M. Patte, Architecte de S.A.S.M. le Prince Palatin, Duc Régnant des Deux-Ponts, Paris, Rozet, 1769.

Piasenza, P., Rapimenti, polizia e rivolta: un conflitto sull'ordine pubblico a Parigi nel 1750, Quaderni Storici, 1987, 64-1, pp. 129-150.

Piasenza, P., Polizia e città. Strategie d'ordine, conflitti e rivolte a Parigi tra sei e settecento, Bologne, Il Mulino, 1990.

Rancière, J., La Mésentente, Paris, Galilée, 1995.

Roche, D., Les pratiques de l'écrit dans les villes françaises du XVIII ${ }^{\mathrm{e}}$ siècle, in Chartier, R. (dir.), Pratiques de la lecture, Paris, Payot, 1985, 1993, pp. 201-229.

Roche, D., La France des Lumières, Paris, Fayard, 1998.

Roche, D., Darnton, R., Revolution in Print. The Press in France, 1775-1800, The New York Public Library, 1989.

Roussel, D., Violences et passions dans le Paris de la Renaissance, Seyssel, Champ Vallon, 2012.

Roussel, D., «Comme en la plus belle forest du monde» ? Discours de l'insécurité et insécurité des discours à Paris au XVI ${ }^{\mathrm{e}}$ siècle, in Belleguic, T., Turcot, L. (dir.), Les histoires de Paris (XVI ${ }^{e}$-XVIII ${ }^{e}$ siècles), Paris, Hermann, 2013, t. 1., pp. 31-51.

Thuau, E., Raison d'État et pensée politique à l'époque de Richelieu, Paris, Albin Michel, 2000.

Versini, L. (éd.), Diderot, t. V, Correspondance, Paris, Robert Laffont, 1997.

Vidoni, N., La Lieutenance générale de police et l'espace urbain parisien (1667-1789). Expériences, pratiques et savoirs, thèse de l'Université de Provence, 2011, 2 vol.

Vittu, J.-P., Journal des Savants, notice 710, in Sgard, J. (dir.), Dictionnaire des Journaux, 1600-1789, Paris, Universitas, 1991, pp. 645-654.

Wittman, R., Politique et publications sur Paris au XVIII ${ }^{\mathrm{e}}$ siècle, Histoire urbaine, 2009-1, 24, pp. 9-32. 